\title{
Eccentric Macular Hole Formation Following Successful Macular Hole Surgery
}

\section{Jasmin Zvorničanin, Edita Zvorničanin, Damir Husić \\ University Clinical Center Tuzla, Department of \\ Introduction} Ophthalmology, Tuzla, Bosnia and Herzegovina

Correspondence:

zvornicanin_jasmin@hotmail.com

Tel.: + 38761134874

Fax.: + 38735303250

Received: 4 October 2019; Accepted: 28 December 2019

\begin{abstract}
Objective. Postoperative eccentric macular hole formation is a rare complication after vitrectomy with internal limiting membrane peeling for a macular hole. We report a rare case of late eccentric macular hole formation following successful macular hole surgery. Case Report. A 73-year old woman was referred with a long standing full thickness macular hole in both eyes. The patient underwent sequential cataract surgery, 23 gauge sutureless pars plana vitrectomy with internal limiting membrane peeling and inverted flap in both eyes. Control examination six months after the surgery revealed best corrected visual acuity of 20/40 in both eyes, complete closure of the macular hole, with retinal atrophy in both eyes. Control examination eighteen months after the surgery revealed a stable finding in the right eye and an eccentric temporal macular hole in the left eye. The patient was closely observed for one year with no signs of visual acuity reduction or macular hole changes. Conclusion. Postoperative eccentric macular holes may occur after a long follow up period. Most cases remain stable for a long period of time and do not require further surgical intervention. This case highlights the need for close monitoring of patients after macular hole surgery with internal limiting membrane peeling.
\end{abstract}

Key Words: OCT - Vitrectomy - Complication.
Macular holes $(\mathrm{MH})$ are defined as a retinal break in the fovea involving the partial to complete dehiscence of the neural retinal layers. Surgical treatment options for $\mathrm{MH}$ include: pars plana vitrectomy and intravitreal ocriplasmin application (1). Vitrectomy, with internal limiting membrane (ILM) peeling and gas tamponade has become a routine technique for $\mathrm{MH}$ surgery, with a postoperative closure rate of $90 \%(1,2)$. However, macular ILM peeling has been associated with complications, including trauma to the retina, macular phototoxicity, focal retinal hemorrhages, macular edema, postoperative swelling of the arcuate retinal nerve fiber layer (SANFL) followed by dissociated optic nerve fiber layer (DONFL), foveal displacement and, in extremely rare cases, iatrogenic eccentric full-thickness retinal breaks (2).

We report a rare case of late eccentric macular hole formation following successful macular hole surgery.

\section{Case Report}

A 73-year old woman was referred with low vision in both eyes, noted 1 year previously. Clinical examination revealed a best corrected distance visual acuity (BCDVA) of 20/400 in the right eye and 20/200 in the left one. Anterior segment examination of both 
eyes showed moderate cataracts. The intraocular pressure measured was $15 \mathrm{mmHg}$ bilaterally. Fundus examination of both eyes revealed the presence of full thickness macular holes (FTMH). Optical coherence tomography (OCT) confirmed the presence of FTMH in both eyes, with a basal hole diameter of $278 \mu \mathrm{m}$ in the right eye and $334 \mu \mathrm{m}$ in the left (Panel A). OCT images were acquired with the Cirrus HD-OCT (Carl Zeiss
Meditec, Dublin CA) using the Smart HD Cross Scan Pattern, which captures and averages $100 \mathrm{~B}$-scan images with automatic centering at the fovea or region of interest. The patient was scheduled for surgical treatment of both eyes.

Two months after the initial examination, the patient underwent sequential cataract surgery with 23 gauge sutureless pars plana vitrectomy of both eyes in general an-

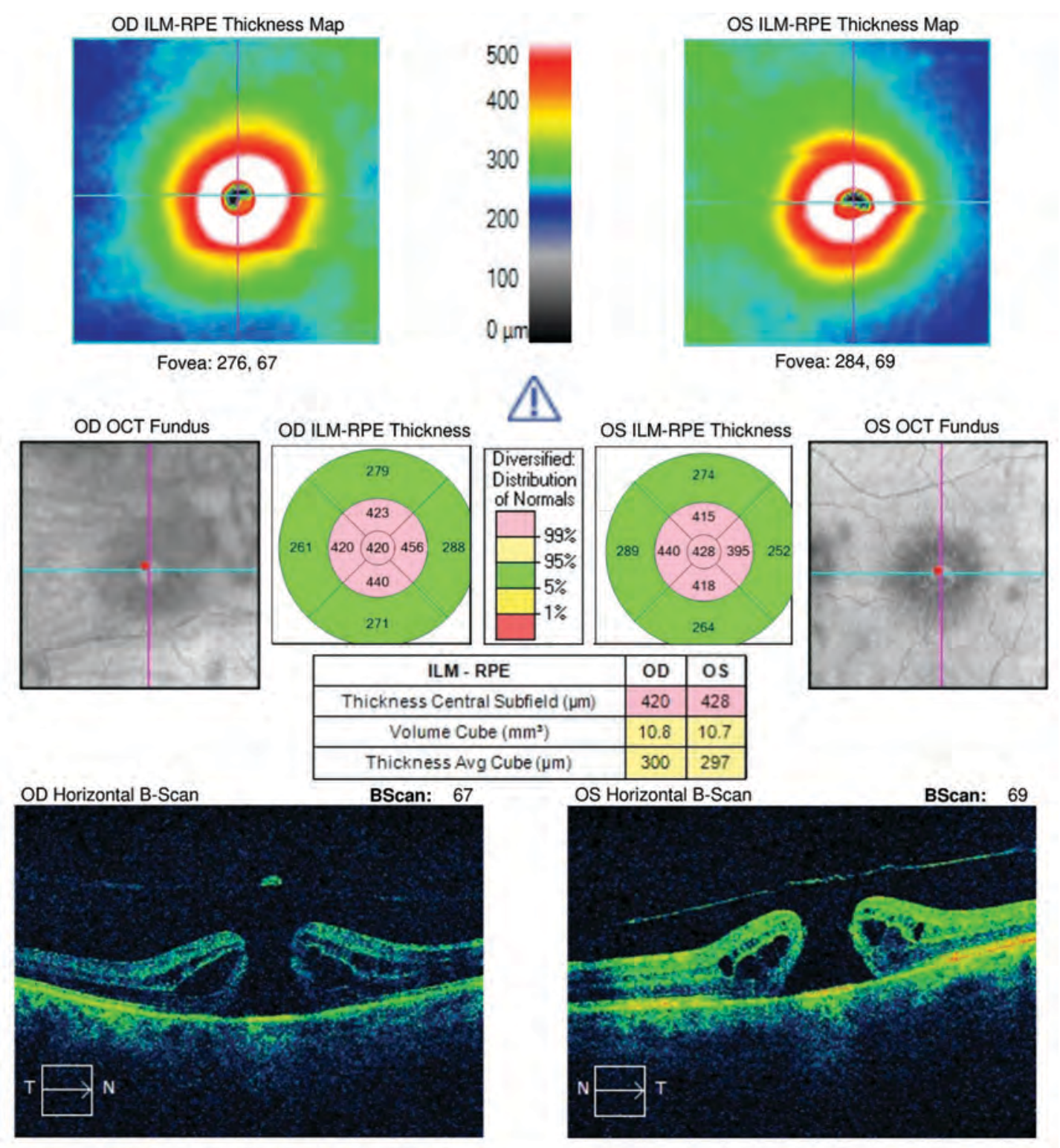

Panel A. Pre-operative OCT showing bilateral FTMH, with a basal hole diameter of $278 \mu \mathrm{m}$ in the right eye and $334 \mu \mathrm{m}$ in the left eye. 
esthesia. The ILM peeling was assisted with Brilliant Peel $^{\circledR}$ (Fluoron, Geuder AG Heidelberg, Germany) and performed with ILM forceps (Grieshaber, Alcon Laboratories, Inc., Fort Worth, TX). The ILM was grasped with ILM forceps and peeled off in a circular fashion for approximately 2 disc diameters around the macular hole. The ILM was not removed completely from the retina but was left attached to the edges of the MH. Periph- eral parts of the ILM were trimmed with the vitrector, and the central part was used as a multilayer ILM flap to cover the MH. Having in mind the duration and the size of the $\mathrm{MH}$, we subsequently enlarged the ILM peeling area $360^{\circ}$ around the macula up to the vascular arcades. We used a gas tamponade with 18\% Sulfur Hexafluoride (SF6), and advised postoperative face-down positioning for a minimum of 3 days. Both sur-

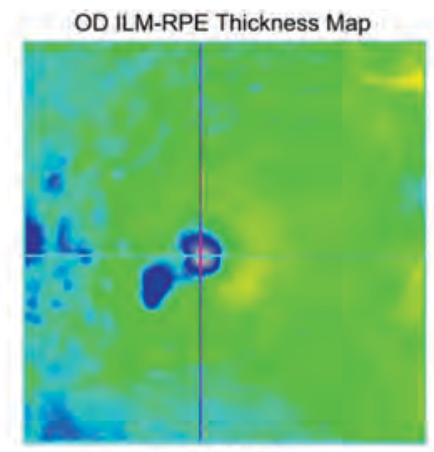

Fovea: 226,69
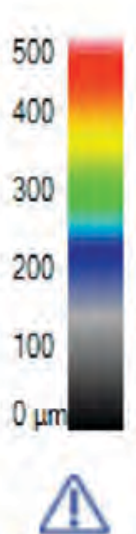

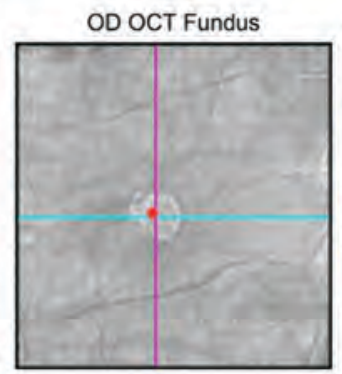
OD ILM-RPE Thickness
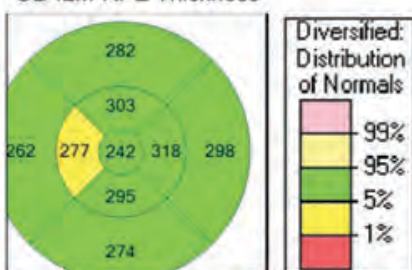

OS ILM-RPE Thickness

Fovea: 250,70

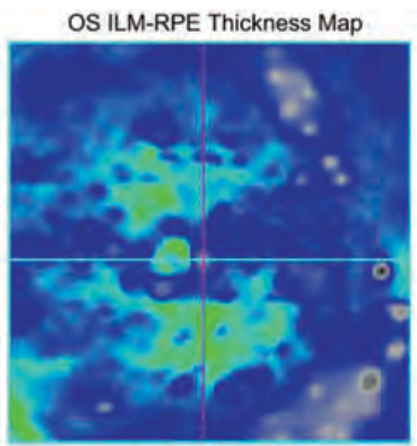

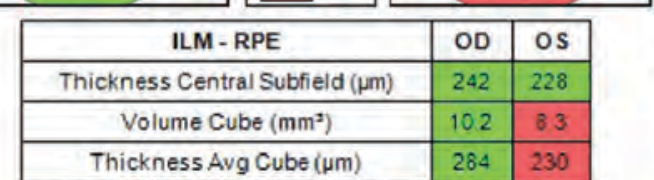

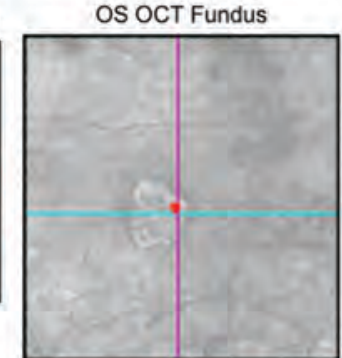

BScan: 69
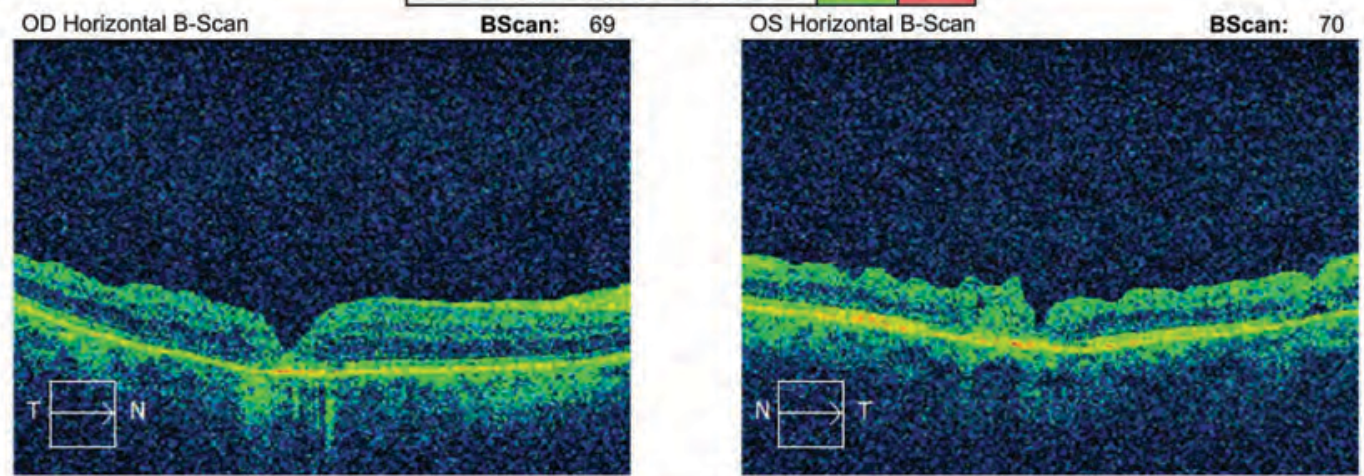

Panel B. Postoperative OCT 6 months after the surgery showing the closure of the FTMH in both eyes, with retinal gliosis in the fovea, resulting in an almost completely absent IZ with discontinuous junction of the IS/OS line and inner retina nerve fiber layer dimpling, more pronounced in the left eye. 
geries were performed by the same surgeon under the same operative conditions, with the same postoperative regimen.

Gradual postoperative visual improvement was noted, and a follow-up examination six months after the surgery revealed BCDVA of 20/40 in both eyes, and best corrected near visual acuity of Jaeger 4 in both eyes. Fundus examination showed complete resolution of FTMH in both eyes, with as- sociated macular atrophy. Control OCT examination showed complete closure of the FTMH in both eyes, with retinal gliosis in the fovea, resulting in an almost completely absent interdigitation zone (IZ) and a discontinuous junction between the photoreceptor inner and outer segment (IS/OS) line, more pronounced in the left eye. Furthermore, inner retina nerve fiber layer dimpling was present in both eyes, more pronounced

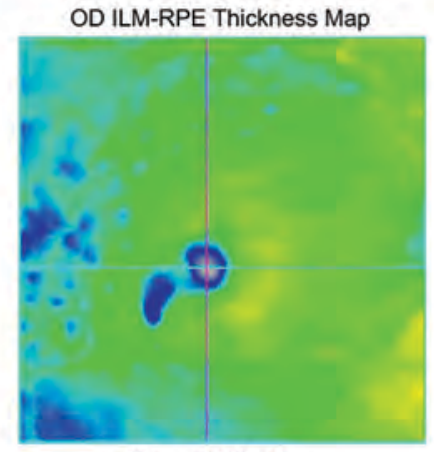

Fovea: 237,73
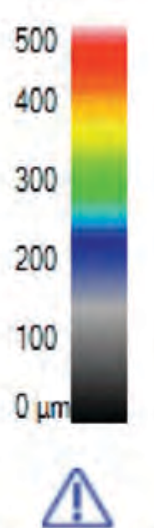

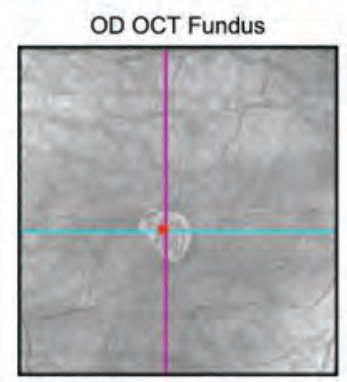

OD ILM-RPE Thickness
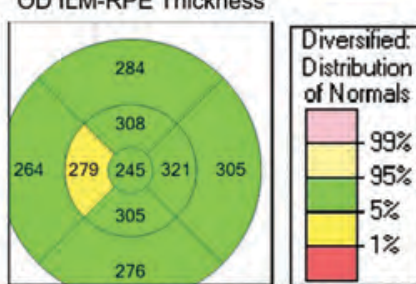

OS ILM-RPE Thickness
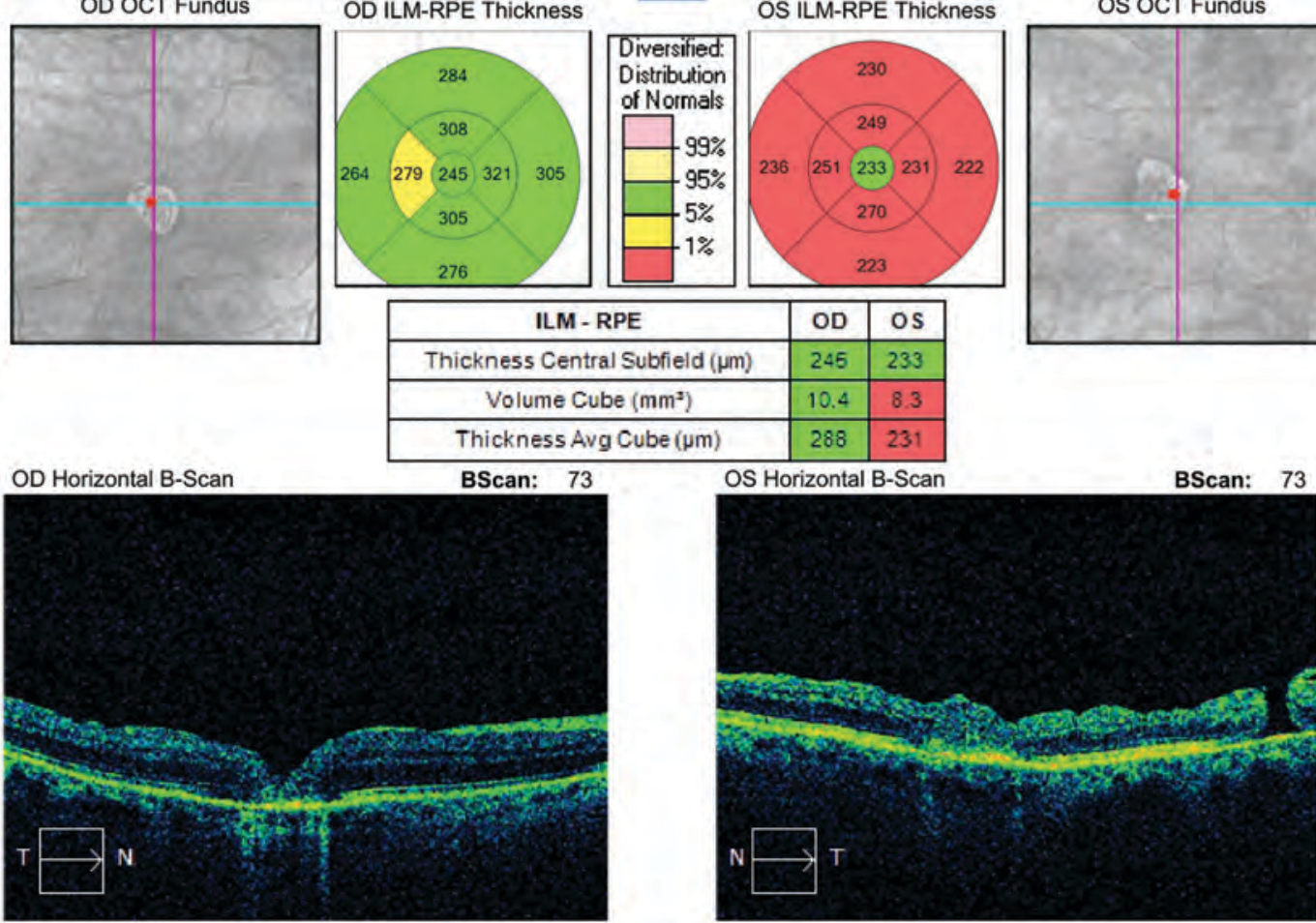

Panel C. Control OCT 18 months after the surgery showing a stable finding in the macula of the right eye, and the eccentric $\mathrm{MH}$ temporal to the fovea in the left eye. 
in the left eye, indicating possible surgical trauma (Panel B). A follow-up examination 18 months after the surgery revealed the same BCDVA in both eyes, a stable finding in the macula of the right eye, while an eccentric temporal $\mathrm{MH}$ was found in the left eye (Panel C). No additional surgery was suggested at that time. Follow-up examinations, three, six and twelve months following the occurrence of the eccentric $\mathrm{MH}$, showed preserved BCDVA and stable OCT findings.

\section{Discussion}

The presence of an eccentric $\mathrm{MH}$ is a rare finding in patients after ILM peeling, reported in less than $0.6 \%$ of cases $(2,3)$. Several theories have been proposed concerning the pathogenesis implicated, including induced surgical trauma, the use of intraocular dyes during the peeling, removal of the ILM layer itself inducing a weakening of retinal glial structures, and the contraction of the residual ILM $(3,4)$. Tangential ILM edge contracture may explain the delayed occurrence of the eccentric $\mathrm{MH}$ and its location at the edge of the ILM peeling area. However, we found no signs of any epimacular membrane proliferation, which could be the cause of eccentric $\mathrm{MH}$ formation $(3,4)$. Nevertheless, in our case OCT images presented significant retinal atrophy with inner retina nerve fiber layer dimpling, which suggest that mechanical surgical trauma probably played the most important role in the pathogenesis of this eccentric $\mathrm{MH}$ (4).

A postoperative $\mathrm{MH}$ may either be lamellar or full-thickness and located centrally, paracentrally, or eccentrically (4). Eccentric MHs close to the fovea are linked to poor visual prognosis as compared to more distant eccentric MHs $(3,4)$. Regarding further intervention, it is necessary to consider the $\mathrm{BCD}$ $\mathrm{VA}$, the location of the $\mathrm{MH}$, and its evolution in terms of size (3). In rare cases, an eccentric $\mathrm{MH}$ may enlarge and lead to central $\mathrm{MH}$ re- opening $(2,4)$. In our case, no further intervention was recommended since the BCDVA and fundus findings remained stable.

\section{Conclusion}

It is important to make a detailed postoperative examination in all patients after vitrectomy with ILM peeling, regardless of the BCDVA and stable central macular finding.

What Is Already Known on this Topic:

Internal limiting membrane peeling is the standard of treatment for macular holes. Postoperative eccentric macular hole formation is a rare complication after vitrectomy with internal limiting membrane peeling for a macular hole.

\section{What this Case Adds:}

Eccentric macular holes may occur long after the internal limiting membrane peeling. Our case demonstrates how bilateral macular hole surgery in one patient can result in different postoperative findings in both eyes. Eccentric macular hole cases can be successfully managed conservatively by observation.

Authors' Contributions: Conception and design: JZ and EZ; Acquisition, analysis and interpretation of data: JZ, EZ and DH; Drafting the article: JZ and EZ; Revising it critically for important intellectual content: JZ and EZ; Approved final version of the manuscript: $\mathrm{JZ}, \mathrm{EZ}$ and DH.

Conflict of Interest: The authors declare that they have no conflict of interest.

\section{References}

1. Bikbova G, Oshitari T, Baba T, Yamamoto S, Mori K. Pathogenesis and Management of Macular Hole: Review of Current Advances. J Ophthalmol. 2019;2019:3467381.

2. Asencio-Duran M, Manzano-Muñoz B, VallejoGarcía JL, García-Martínez J. Complications of Macular Peeling. J Ophthalmol. 2015;2015:467814.

3. Sandali O, El Sanharawi M, Basli E, Lecuen N, Bonnel S, Borderie V, et al. Paracentral retinal holes occurring after macular surgery: incidence, clinical features, and evolution. Graefes Arch Clin Exp Ophthalmol. 2012;250:1137-42.

4. Brouzas D, Dettoraki M, Lavaris A, Kourvetaris D, Nomikarios N, Moschos MM. Postoperative eccentric macular holes after vitrectomy and internal limiting membrane peeling. Int Ophthalmol. 2017;37:643-8. 